\title{
Paradoxical air embolism due to electrosurgical vaporization during hysteroscopic myomectomy
}

\author{
Young-Seok Lee, Soo-Eun Choi, Sung-Wook Park, and Keon-Sik Kim \\ Department of Anesthesiology and Pain Medicine, College of Medicine, Kyung Hee University, Seoul, Korea
}

Air emboli can arterialize and result in paradoxical air embolism (PAE). In most cases there is a right to left shunt, and for this to occur during a hysteroscopic surgery is extremely rare. This case report describes a PAE found in the left heart during hysteroscopic myomectomy without an anatomical right to left shunt.

A 29-yr-old woman (height $170 \mathrm{~cm}$, weight $62 \mathrm{~kg}$, American Society of Anesthesiologists I) was seen at our gynecologic clinic due to a known uterine myoma. She had no specific medical history, and had given birth to a preterm baby of 24 weeks by cesarean section under general anesthesia 6 months earlier. There were no abnormal findings in the preoperative examination and she had no risk factors for cardiac or pulmonary disease. For maintenance during the operation, $\mathrm{O}_{2} 2.0 \mathrm{~L} / \mathrm{min}, \mathrm{N}_{2} \mathrm{O} 2.0 \mathrm{~L} / \mathrm{min}$, and desflurane $4.0-6.0$ vol\% was used. Though at the beginning of the surgery vital signs were stable, after $15 \mathrm{~min}$ systolic and diastolic blood pressure decreased to $80-100 \mathrm{mmHg}$, and 40-60 $\mathrm{mmHg}$ respectively. Arterial blood gas analysis (ABGA) revealed that the $\mathrm{pH}$ was 7.384, $\mathrm{PaO}_{2} 271.9 \mathrm{mmHg}, \mathrm{PaCO}_{2} 29.8 \mathrm{mmHg}$, hemoglobin $6.9 \mathrm{~g} /$ dl, Hct $20 \%$. As systolic blood pressure continued to drop to $80-$ $90 \mathrm{mmHg}$, diastolic blood pressure to $40-50 \mathrm{mmHg}$, and mean arterial pressure (MAP) to $60-65 \mathrm{mmHg}, 10 \mathrm{mg}$ of ephedrine was administered, while 2 units of packed RBC transfusion was initiated. In order to continue measuring blood pressure and arterial blood gas, a 20 gauge catheter was inserted in the left radial artery. The results of the ABGA after the radial artery cannulation were $\mathrm{pH}$ 7.336, $\mathrm{PaO}_{2} 291.1 \mathrm{mmHg}, \mathrm{PaCO}_{2} 35.9$ mmHg, and $\mathrm{P}_{\mathrm{ET}} \mathrm{CO}_{2} 25 \mathrm{mmHg}$. Though $\mathrm{P}_{\mathrm{ET}} \mathrm{CO}_{2}$ decreased only slightly, the decreasing blood pressure and signs of increase in $\mathrm{PaCO}_{2}$ gave rise to suspicions of pulmonary embolism, so $\mathrm{N}_{2} \mathrm{O}$ was discontinued and oxygen and medical air were both maintained at $2.0 \mathrm{~L} / \mathrm{min}$. Ninety minutes after the beginning of the surgery, the MAP suddenly dropped to $60 \mathrm{mmHg}$ and the $\mathrm{P}_{\mathrm{ET}} \mathrm{CO}_{2}$ could not be detected (having decreased to $0 \mathrm{mmHg}$ ). The ABGA initiated at this point and showed $\mathrm{pH}$ 7.149, $\mathrm{PaO}_{2}$ $65.4 \mathrm{mmHg}$, and $\mathrm{PaCO}_{2} 35.9 \mathrm{mmHg}$. Because the changes in hemodynamics coupled with the results of the ABGA lead to suspicions of pulmonary embolism, $\mathrm{O}_{2}$ supply was kept at $100 \%$ and positive pressure ventilation was initiated. Transthoracic echocardiography (TTE) was used to confirm an air embolism in the left heart (Fig. 1). The lung perfusion scan performed after the surgery revealed multiple non-matched small non-

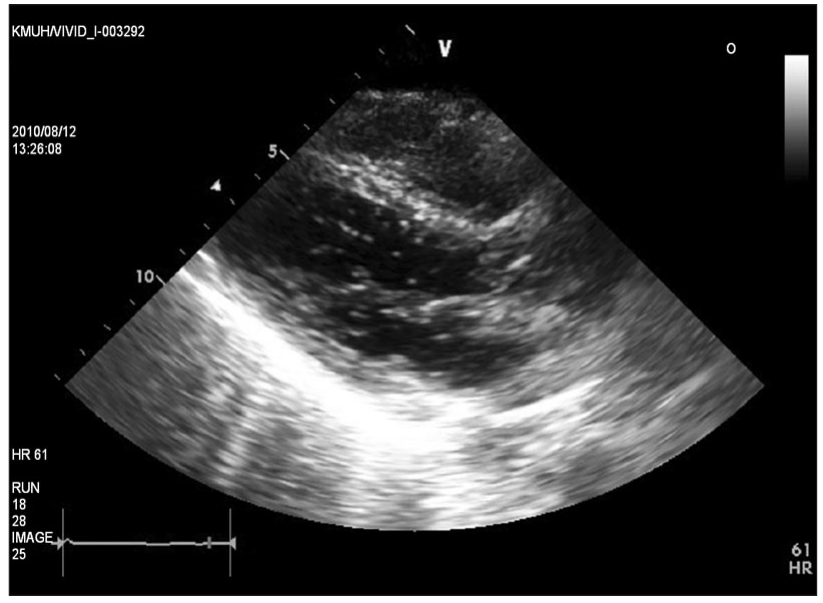

Fig. 1. Transthoracic echocardiogram in the parasternal long axis view at the operation room shows air bubbles in the left heart.

Corresponding author: Keon-Sik Kim, M.D., Department of Anesthesiology and Pain Medicine, Kyung Hee Medical Center, 1, Hoegi-dong, Dongdaemun-gu, Seoul 130-702, Korea. Tel: 82-2-958-8589, Fax: 82-2-958-8580, E-mail: keonsik@hanafos.com

(c) This is an open-access article distributed under the terms of the Creative Commons Attribution Non-Commercial License (http:// creativecommons.org/licenses/by-nc/3.0/), which permits unrestricted non-commercial use, distribution, and reproduction in any medium, provided the original work is properly cited. 
segmental perfusion defects in both lungs indicating pulmonary embolism. Two days after the surgery, the patient returned from the ICU to the general ward, and a TTE investigation with an agitate saline test was performed to exclude any possible intracardiac shunt. The chest computed tomography (CT) revealed no evidence of thromboembolism.

Gas embolism occurs when large quantities of gas from an open noncollapsible vein or artery enter systemic circulation. This gas embolism rarely arterializes and becomes a PAE to be confirmed by TTE. In this case we used normal saline as a uterine distension medium to remove air in all intravenous lines and hysteroscopic instruments. A tenaculum was used to minimize cervical exposure after cervical dilation and a continuous outflow system was used to refresh the bubbles and debris in the uterus. Thus, we were able to eliminate the possibility of gas entering from an outside source, and suspect that the highly soluble gases, such as hydrogen, carbon monoxide, and carbon dioxide produced during electrosurgical vaporization of the myoma created an unlikely air entrainment and entered the circulation [1].

There are known possible mechanisms that venous air embolism (VAE) from the right heart can cross over to the left heart to become a PAE. The most common mechanism of PAE is caused by abnormal intracardiac communication, such as ASD, VSD, or patent foramen ovale (PFO). When we had done the TTE test 2 days after the event, we were able to exclude PFO or other intracardiac shunts as causes of the embolism.

Other possible mechanisms of arterialized air embolism include pathological dilatation in intrapulmonary vessels or pulmonary AV malformation in patients suffering from hereditary hemorrhagic telangiectasia or chronic liver disease [2]. These patients have a pulmonary right to left shunt or high sensitivity to contrast in enhanced TTE diagnosis. However, in our patient we discovered no atypical hereditary or genetic disorders and no particular reason to suspect intrapulmonary shunt. Upon TTE, none were found.

In patients without anatomical shunt, transpulmonary passage of venous air emboli could be a cause for the PAE. Typically, pulmonary circulation has filtering capabilities with respect to air emboli. However, paradoxical air emboli may occur, for e.g. emboli are too small to properly filter the lung capillaries [3] or the emboli's total volume is above fixed levels and exceeds the lungs filtration capacity [4]. Lung capillaries have a diameter of approximately $3-15 \mu \mathrm{m}$ and they can filter any dysmorphic emboli with a diameter greater than $14-22 \mu \mathrm{m}$. Although the precise amount of suspected high soluble gases produced by electrosurgical vaporization is unknown, it is very possible that a considerable number of microbubbles smaller than $14-22 \mu \mathrm{m}$ in diameter can form. Data from an animal study suggests that the lung acts as a physiologic filter, which becomes overwhelmed above $0.3 \mathrm{ml} / \mathrm{kg} / \mathrm{min}$ [3], but the exact maximum volume of air filtering capacity in the human lung is not known. In vitro studies have proven that electrosurgical vaporization can produce gas at up to $60 \mathrm{ml} / \mathrm{min}$ [5]. Therefore, the total volume of gas in the patient could have exceeded the lung's filtering capacity and caused the PAE. The transpulmonary passage of venous air emboli can be a pertinent cause for our case.

In conclusion, this case demonstrates that paradoxical emboli may develop during hysteroscopic surgery in patients without an anatomical right to left shunt. Although hysteroscopic surgery is not typically thought to be a high risk procedure for air embolism, this case demonstrates the possibility of air embolism by electrosurgical vaporization.

\section{References}

1. Munro MG, Weisberg M, Rubinstein E. Gas and air embolization during hysteroscopic electrosurgical vaporization: comparison of gas generation using bipolar and monopolar electrodes in an experimental model. J Am Assoc Gynecol Laparosc 2001; 8: 488-94.

2. Hopkins WE, Waggoner AD, Barzilai B. Frequency and significance of intrapulmonary right-to-left shunting in end-stage hepatic disease. Am J Cardiol 1992; 70: 516-9.

3. Butler BD, Hills BA. Transpulmonary passage of venous air emboli. J Appl Physiol 1985; 59: 543-7.

4. Butler BD, Katz J. Vascular pressures and passage of gas emboli through the pulmonary circulation. Undersea Biomed Res 1988; 15 : 203-9.

5. Munro MG, Brill AI, Ryan T, Ciarrocca S. Electrosurgery-induced generation of gases: comparison of in vitro rates of production using bipolar and monopolar electrodes. J Am Assoc Gynecol Laparosc 2003; 10: 252-9. 